\title{
High programmed cell death 1 ligand-1 expression: association with CD8+ T-cell infiltration and poor prognosis in human medulloblastoma
}

\author{
Daiki Murata, MD, ${ }^{1}$ Yohei Mineharu, MD, PhD, ${ }^{1}$ Yoshiki Arakawa, MD, PhD, ${ }^{1}$ Bin Liu, MD, ${ }^{1}$ \\ Masahiro Tanji, MD, ${ }^{1}$ Makoto Yamaguchi, MD, ${ }^{1}$ Ko-ichi Fujimoto, MD, ${ }^{1}$ Nobuyuki Fukui, MD, ${ }^{1}$ \\ Yukinori Terada, MD, ${ }^{1}$ Ryuta Yokogawa, MS, ${ }^{1}$ Maki Yamaguchi, MD, ${ }^{2}$ \\ Sachiko Minamiguchi, MD, PhD, ${ }^{2}$ and Susumu Miyamoto, MD, PhD'
}

Departments of ${ }^{1}$ Neurosurgery and ${ }^{2}$ Diagnostic Pathology, Kyoto University Graduate School of Medicine, Kyoto, Japan

\begin{abstract}
OBJECTIVE Medulloblastoma is a type of malignant tumor arising in the cerebellum. The clinical importance of programmed cell death 1 ligand-1 (PD-L1) expression in medulloblastoma remains unknown. The aim of the present study was to examine the expression of PD-L1 and tumor-infiltrating T cells, and to evaluate their relationships to the prognosis of patients with medulloblastoma.
\end{abstract}

METHODS The authors immunohistochemically analyzed PD-L1 expression and CD3+ and CD8+ lymphocyte infiltrations in tumor specimens from 16 patients with medulloblastoma.

RESULTS High expression of PD-L1 was observed in 9 (56.3\%) of 16 samples studied. High expression of PD-L1 was associated with low infiltrations of CD3+ or CD8+ lymphocytes. Patients with high expression of PD-L1 had shorter progression-free survival and overall survival times than those with low expression ( $p=0.076$ and $p=0.099$, respectively). In addition, patients with high expression of PD-L1 and with low infiltration of CD8+ lymphocytes had a significantly worse outcome, with a 5 -year survival rate of $15 \%$, as compared with the other patients, who had a 5 -year survival rate of nearly $90 \%$ ( $p=0.0048$ for progression-free survival and $p=0.010$ for overall survival).

CONCLUSIONS These findings indicate that PD-L1 expression was associated with a reduced infiltration of CD8+ T cells and poor prognosis in human medulloblastoma.

https://thejns.org/doi/abs/10.3171/2016.11.JNS16991

KEY WORDS medulloblastoma; programmed cell death 1 ligand-1; PD-L1; prognosis; antitumor immunity; lymphocyte; oncology

$\mathrm{M}$ edulloblastoma is one of the most common intracranial pediatric malignancies. The general treatment strategies for medulloblastoma are maximal safe resection followed by chemotherapy and either local or craniospinal radiotherapy, which are selected based on the postoperative evaluation of the prognosis. Although these treatments have improved the prognosis of patients with medulloblastoma, the prognosis of infants and patients with dissemination remains poor. ${ }^{3,4}$ Furthermore, the problem is that radiotherapy causes intellectual complications in long-term survivors. ${ }^{14}$

Programmed cell death-1 (PD-1) is an immunoinhibitory receptor belonging to the $\mathrm{CD} 28$ family that includes cytotoxic T-lymphocyte antigen-4 (CTLA-4), which plays an important role in immune tolerance and immune es- cape for tumor cells. ${ }^{6,12,13}$ Its ligands are programmed cell death ligand-1 (PD-L1; B7-H1) and PD-L2. Whereas PD-L2 is mainly expressed on activated macrophages and dendritic cells, PD-L1 is expressed on tumor cells as well as immune cells. The expression of PD-L1 has been shown to correlate with the prognosis of patients with malignant tumors, although its prognostic value is inconsistent: overexpression of PD-L1 was shown to be associated with worse prognosis in patients with non-small cell lung carcinoma and non-clear cell renal cell carcinoma, ${ }^{2,23}$ whereas it was associated with better prognosis in patients with melanoma, breast cancer, and glioblastoma. ${ }^{7,918}$ Expression of PD-L1 was also shown to be associated with tumor-infiltrating lymphocytes. In ovarian cancer, PD-L1 expression was inversely associated with intraepithelial

ABBREVIATIONS OS = overall survival; PD-L1 = programmed cell death 1 ligand-1; PFS = progression-free survival.

SUBMITTED April 18, 2016. ACCEPTED November 17, 2016.

INCLUDE WHEN CITING Published online May 5, 2017; DOI: 10.3171/2016.11.JNS16991. 
CD8+ lymphocytes, and high expression of PD-L1 and low infiltration of CD8+ lymphocytes were independently associated with poor prognosis in cancer patients. ${ }^{5}$ These findings indicate that an imbalance between the cytotoxic (CD8) and tolerogenic (PD-L1) tumor microenvironment can be an immunological parameter to evaluate the prognosis of malignancies.

Thus, expression of PD-L1 and infiltration of CD8+ lymphocytes can be a fine biomarker for many types of malignancies. However, partly due to the rarity of the disease, these molecular markers have not been tested in medulloblastoma so far. In the present study, we examined the expression of PD-L1 and infiltration of CD3+ and CD8+ lymphocytes with immunohistochemical analysis, and evaluated their correlation with the prognosis of patients with medulloblastoma.

\section{Methods}

\section{Medulloblastoma Cell Lines and Cell Culturing}

Two human medulloblastoma cell lines, D283 Med (HTB-185) and Daoy (HTB-186), were purchased from American Type Culture Collection. All cell lines were established in culture prior to experimentation. Medulloblastoma cell lines were maintained at $37^{\circ} \mathrm{C}, 95 \%$ room air, and 5\% $\mathrm{CO}_{2}$, in DMEM supplemented with $10 \%$ fetal bovine serum and 5\% penicillin-streptomycin $(100 \mathrm{U} / \mathrm{ml}$ penicillin, $100 \mu \mathrm{g} / \mathrm{ml}$ streptomycin). Cell lines were cultured, grown to approximately $80 \%$ confluence, washed in phosphate-buffered saline, and then trypsinized. Cells were subsequently centrifuged, resuspended in serum-free and antibiotic-free DMEM, and counted using a hemocytometer after staining with Trypan blue.

\section{Patient Population and Tumor Specimens}

Formalin-fixed, paraffin-embedded specimens were obtained in 16 patients who underwent primary surgery for medulloblastoma at Kyoto University Hospital between 1987 and 2009 (Table 1). Clinical and pathological characteristics were collected from their medical records and telephone interviews, including age, sex, day of operation, radiation dosage and schedule, chemotherapy regimens, Chang's criteria, Ki-67 index, and survival. After surgery, all patients received radiotherapy and 14 received chemotherapy. The study was approved by the ethics committee of the Kyoto University Hospital.

\section{Immunohistochemical Investigation}

Formalin-fixed, paraffin-embedded specimens were deparaffinized in xylene and dehydrated in ethanol (99\%, $90 \%, 80 \%$, and $70 \%$ ). For antigen retrieval of PD-L1, CD3, and CD8, the specimens were boiled in Tris-EDTA buffer $(\mathrm{pH} 9.0)$ by microwaves. To block endogenous peroxidase activity, the tissues were treated with $100 \%$ methanol containing $0.3 \% \mathrm{H}_{2} \mathrm{O}_{2}$. Nonspecific binding of $\mathrm{IgG}$ was blocked using normal goat serum (Vector). The specimens were incubated with polyclonal rabbit anti-human PD-L1 antibody (LSBio: LS-B480); monoclonal rabbit anti-human CD3 antibody (Abcam); monoclonal mouse anti-human CD8 antibody (Abcam); monoclonal mouse anti-CTNNB1 antibody (BD Transduction Laboratories: ab610154); polyclonal rabbit anti-human SFRP1 (Abcam: ab4193); polyclonal rabbit anti-human NPR3 antibody (Abcam: ab37617); and polyclonal rabbit anti-human KCNA antibody (Abcam: ab32433) overnight at $4^{\circ} \mathrm{C}$. Then the sections were incubated with goat anti-mouse and anti-rabbit secondary antibody (EnVision+ Dual Link System-HRP, Dako). Signals were generated by incubation with 3,3'-diaminobenzidine (Vector). Specimens were counterstained with hematoxylin and observed under a microscope. A section of normal placenta tissue was used as positive control.

\section{Evaluation of Immunohistochemical Staining}

The expression of PD-L1 was evaluated according to the intensity of the staining. We scored the findings as follows: 0 , negative; 1 , very weak expression; 2 , moderate expression; and 3, strongest expression. The scores of 0 or 1 were defined as the low-expression group, and the scores of 2 or 3 were defined as the high-expression group. Two independent observers examined the immunohistochemical slides without any prior information regarding the clinical history of the patients. To objectively validate the assessment, we measured the intensity of the staining by using ImageJ software. The red-green-blue (RGB) method was used, and red color intensity was calculated in the region of interest in the slides with higher magnification $(\times 400) .{ }^{21}$ Five regions of interest $(100 \times 100$ square pixels for each) were randomly selected in the nucleus area, and a mean pixel intensity value was used to quantitatively assess the staining intensity. The pixel intensity values ranged from 0 to 255 , wherein 0 represents the darkest shade of the color and 255 represents the lightest shade of the color as standard. We set a cutoff pixel intensity value of 130 , and a mean pixel intensity of $>130$ was regarded as low expression.

The infiltration of CD3+ lymphocyte and CD8+ lymphocyte was evaluated according to counts of the number of cells after examination of the slides by using at least 3 different high-power fields (maximum, $\times 40$ objective and $\times 10$ eyepiece). Two independent observers examined the specimens by using the same method as above. The number of cells that stained positively for CD3 and CD8 was recorded. The result was interpreted as low infiltration when there were $\leq 5$ cells per 3 fields, and as high infiltration when there were $>5$ cells per 3 fields.

\section{Molecular Subtypes}

Molecular subtypes were determined by immunohistochemical studies because staining for DKK1 (WNT), SFRP1 (SHH), NPR3 (Group 3), and KCNA1 (Group 4) has been shown to reliably and uniquely classify formalin-fixed medulloblastomas in approximately $98 \%$ of patients. ${ }^{11}$

\section{Results}

\section{Expression of PD-L1, CD3, and CD8 in Medulloblastoma Specimens}

The expression of PD-L1 was analyzed in 16 medulloblastoma specimens by using immunohistochemical studies. High expression (score of 2 or 3) of PD-L1 was 
TABLE 1. Characteristics of 16 patients with medulloblastoma

\begin{tabular}{|c|c|c|c|c|}
\hline \multirow[b]{2}{*}{ Characteristic } & \multirow[b]{2}{*}{ Total } & \multicolumn{2}{|c|}{ Tumor Type } & \multirow{2}{*}{$\begin{array}{c}p \\
\text { Value }\end{array}$} \\
\hline & & PD-L1(H)+CD8(L) & Others & \\
\hline Total patients & 16 & 7 & 9 & \\
\hline Median age in yrs, range & $13.5,2-43$ & $16,6-43$ & $8,2-24$ & 0.096 \\
\hline$\leq 3$ & $1(6.3)$ & $0(0)$ & $1(11.1)$ & 1 \\
\hline$\leq 16$ & $10(62.5)$ & $4(57.1)$ & $6(66.7)$ & 1 \\
\hline Male & $11(68.7)$ & $5(71.4)$ & $6(66.7)$ & 1 \\
\hline \multicolumn{5}{|l|}{ Chang grade } \\
\hline M0-1 & $13(81.2)$ & $6(85.7)$ & $7(77.8)$ & 1 \\
\hline M2 & $3(18.8)$ & $1(14.3)$ & $2(22.2)$ & \\
\hline M3 & $0(0)$ & $0(0)$ & $0(0)$ & \\
\hline High risk & $5(31.3)$ & $1(14.3)$ & $4(44.4)$ & 0.197 \\
\hline Surgery, GTR/partial & $12 / 4$ & $6 / 1$ & $6 / 3$ & 0.584 \\
\hline Radiation & $16(100)$ & $7(100)$ & $9(100)$ & 1 \\
\hline Mean Ki-67, range & $20.0,7.4-80.0$ & $20.7,7.4-50.0$ & $20.0,15.9-80.0$ & 0.572 \\
\hline \multicolumn{5}{|l|}{ Molecular subtypes* } \\
\hline WNT & $1(6.3)$ & $0(0)$ & $1(11.1)$ & 0.369 \\
\hline $\mathrm{SHH}$ & $4(25.0)$ & $3(50.0)$ & $1(11.1)$ & \\
\hline Group 3 & $3(18.8)$ & $1(16.7)$ & $2(22.2)$ & \\
\hline Group 4 & $7(43.8)$ & $2(33.3)$ & $5(55.6)$ & \\
\hline
\end{tabular}

observed in 9 cases (56.3\%), and low expression (score of 0 or 1) was observed in $7(43.8 \%)$ cases (representative cases are shown in Fig. 1). Quantitative assessment of intensity of staining with a mean pixel intensity value was in complete agreement with the qualitative assessment: all of the high-expression cases showed a mean pixel intensity of $\leq 130$ and all of the low-expression cases showed a mean pixel intensity of $>130$. Tumor-infiltrating CD3+ and CD8+ lymphocytes were also examined. High infiltration of CD3+ lymphocytes was observed in 7 (43.8\%) cases and high infiltration of CD8+ lymphocytes was observed in $6(37.5 \%)$ cases (representative cases are shown in Fig. 1).

\section{Correlation Between PD-L1, CD8, and CD3 Expressions and Clinical Characteristics}

The average age at diagnosis for the 16 patients was 13.5 years (range $2-43$ years). The mean follow-up period was 79.8 months (range 7.2-171.0 months), and 7 (43.8\%) patients died of the disease (Table 1). Clinical characteristics were compared according to the expression of PD$\mathrm{L} 1, \mathrm{CD} 8$, or CD3. There was no significant difference of age, sex, Chang's criteria, Ki-67, and molecular subtypes (WNT, SHH, Group 3, and Group 4) according to the expression of these markers.

\section{High PD-L1 and low CD8 Expressions: Correlation With Poor Progression-Free Survival and Overall Survival}

The Kaplan-Meier survival curve demonstrated that both the progression-free survival (PFS) and overall sur- vival (OS) in patients with high PD-L1 expression was worse than in those with low expression (Fig. 2A and B). The log-rank test showed marginally significant difference $(\mathrm{p}<0.1)$ in both PFS $(\mathrm{p}=0.076)$ and OS $(\mathrm{p}=0.099)$. Seven of 9 specimens with high PD-L1 expression showed low infiltration of CD8+ lymphocytes, and 4 of 7 specimens with low PD-L1 expression showed high infiltration of CD8+ lymphocytes. Patients with high infiltration of CD8+ lymphocytes showed better survival than those with low CD8 expression, although it was not statistically significant (Fig. 2C and D). Similar survival trends were observed according to infiltrations of CD3+ lymphocytes (Fig. 2E and F).

Of note, the combination of PD-L1 expression and CD8+ lymphocyte infiltration improved the risk stratification: patients with high PD-L1 expression and low CD8+ lymphocyte infiltration had a significantly worse prognosis than the other patients for both PFS $(\mathrm{p}=0.0048)$ and OS $(p=0.010)$ (Fig. 3). Stratified analysis using pediatric cases 16 years of age and younger also showed a significant difference $(p=0.0088$ for PFS and $p=0.0276$ for OS). On the other hand, Chang's criteria or Ki-67 index was not significantly associated with PFS or OS. Clinical and other pathological characteristics including Chang's criteria, Ki-67 index, and molecular subtypes (WNT, SHH, Group 3, and Group 4) were not different between patients with high PD-L1 expression and low CD8+ lymphocyte infiltration and the other patients, although age at onset was older in patients with high PD-L1 expression and low CD8+ lymphocyte infiltration (Table 1). 


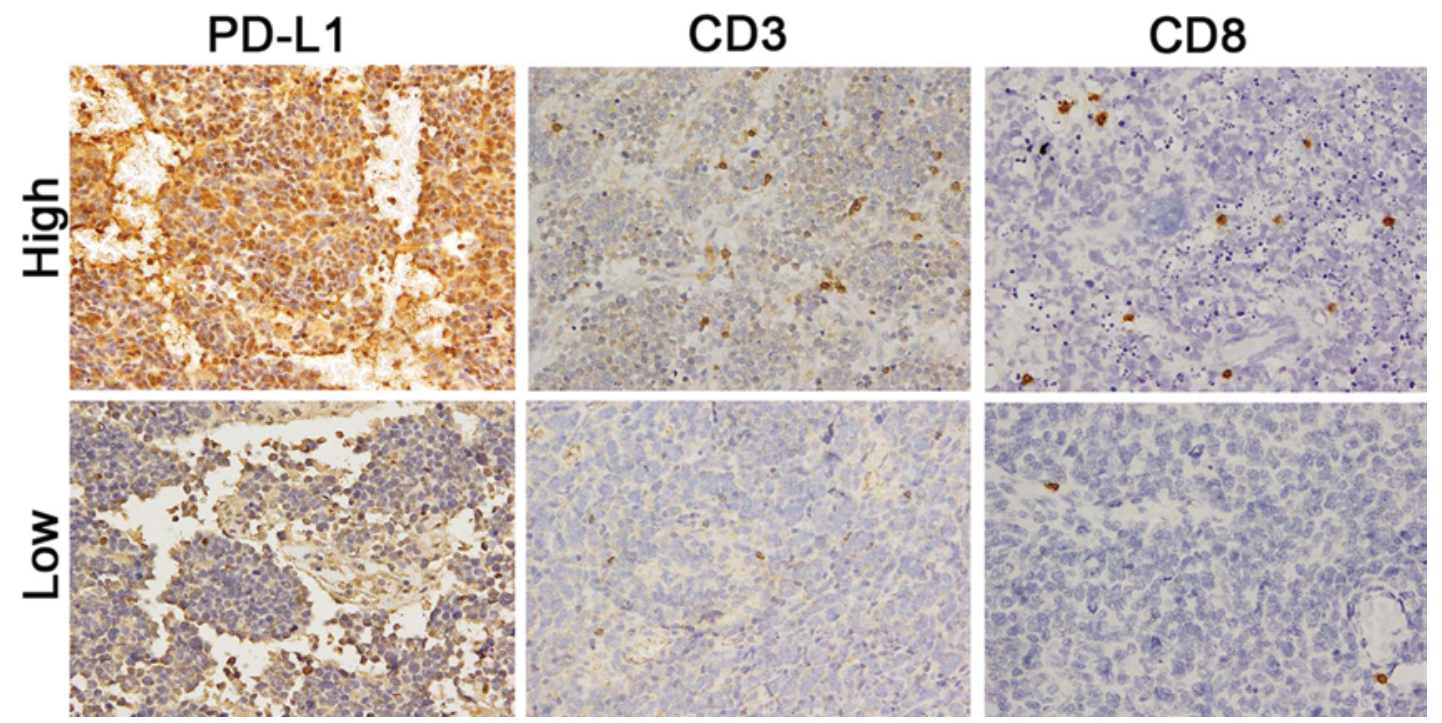

FIG. 1. Photomicrographs of sections used for immunohistochemical analysis and evaluation of PD-L1, CD3, and CD8 in human medulloblastoma specimens. Expression of PD-L1 and infiltration of CD3+ and CD8+ lymphocytes were analyzed by immunohistochemical studies in tumor specimens obtained in patients with medulloblastoma. Representative cases of high and low expression and/or infiltration are shown. Trypan blue, hematoxylin counterstain; original magnification $\times 400$.

\section{Discussion}

Our data demonstrated that high PD-L1 expression was associated with lower CD8+ lymphocyte infiltration and worse prognosis in medulloblastoma. Infiltrations of CD3+ lymphocytes did not show a strong correlation either with PD-L1 or the prognosis of patients with medulloblastoma. These findings suggest that PD-L1 expressed on medulloblastoma may shift the balance of T lymphocytes (CD3+) into lower cytotoxic (CD8+) lymphocyte and higher regulatory $\mathrm{T}$ lymphocytes, leading to tumor progression. Interestingly, the combination of PD-L1 and CD8 assessment was more accurate in distinguishing patients with good prognosis from those with poor prognosis.

Nearly half of the patients showed high PD-L1 expression and low CD8+ lymphocyte infiltration, and they had a 5-year survival rate of as low as $15 \%$. In contrast, the other half of patients had a 5-year survival rate of approximately $90 \%$. These findings suggest that immunohistochemical analysis of PD-L1 and CD8, which reflect an antitumor immune microenvironment, could be a good biomarker for medulloblastoma, as is the case in other cancers. ${ }^{5,18}$ Considering that the shortest 5-year survival stratified by a single genetic alteration is $40 \%-60 \%$, risk stratification by PD-L1 expression and CD8+ lymphocyte infiltration seems to be very powerful. Recently, a correlation between the expression of PD-L1 and the prognosis was demonstrated in mouse models of medulloblastoma. ${ }^{16}$ Further combined analysis of the expressions of PD-L1 and CD8 in these models will be worthwhile to confirm our result.

Medulloblastoma has recently been recognized as a heterogeneous disease that can be divided into 4 subtypes according to genetic architecture: Wnt, SHH, Group 3, and Group 4.,19 The prognosis of patients with medulloblastoma differs among these genetic subtypes. The Wnt subtype shows the most favorable prognosis among these subtypes, rendering a 5-year survival rate of $90 \%$. Group 3 medulloblastoma, whose hallmark is MYC amplification, is the most aggressive subtype, showing a 5-year survival rate of $40 \%-60 \%$. Other subtypes have an intermediate prognosis, with a 5-year survival rate of approximately $75 \%$. It is therefore suspected that PD-L1 expression was different between these molecular subtypes, and that high PD-L1 expression with low CD8 cell infiltration might be more frequently observed in Group 3 medulloblastomas; however, we could not find such correlations.

The PD-L1 protein level in tumor samples from patients with glioblastoma has been reported to be correlated with PTEN loss..$^{15}$ Similarly, genetic alterations in medulloblastoma may have influence on PD-L1 expression. Recently, a combination of genetic markers was shown to improve prognostic risk stratification for medulloblastoma. ${ }^{19}$ In the SHH subtype, GLI2 amplification was associated with worse survival. In the Group 3 subtype (MYC amplification), isochromosome of $17 \mathrm{q}$ was associated with worse survival. Thus, $M Y C$ or GLI2, a genetic indicator for poor prognosis, might have influence on PD-L1 expression. Although it is difficult to test this assumption in our small study population, further study is necessary to see the relationship between PD-L1 expression and the gene signature of medulloblastoma.

Anti-PD-L1 antibody has emerged as an effective immunotherapy that is effective in various kinds of cancer by inducing potent antitumor immune responses. ${ }^{8,17,20,24} \mathrm{Be}-$ cause high expression of PD-L1 and reduced infiltration of cytotoxic lymphocytes were associated with poor prognosis in medulloblastoma, anti-PD-L1 blocking antibody could be a potential treatment option for patients with medulloblastoma, especially for those with poor prognosis. On the other hand, mutation-targeted treatments for specific subtypes of medulloblastoma have been studied 
A

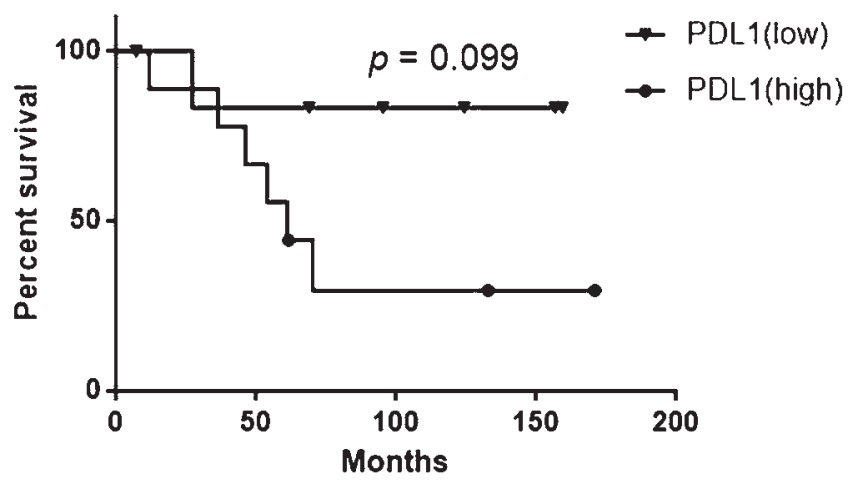

C
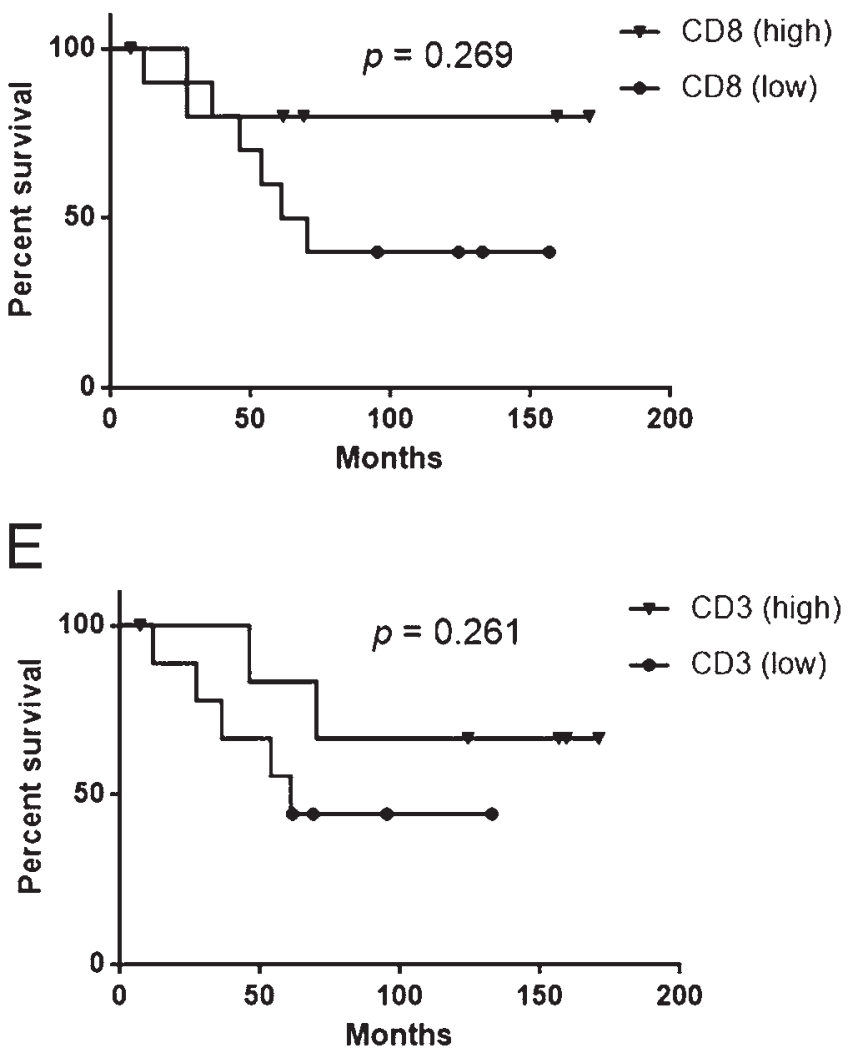

B Progression free survival

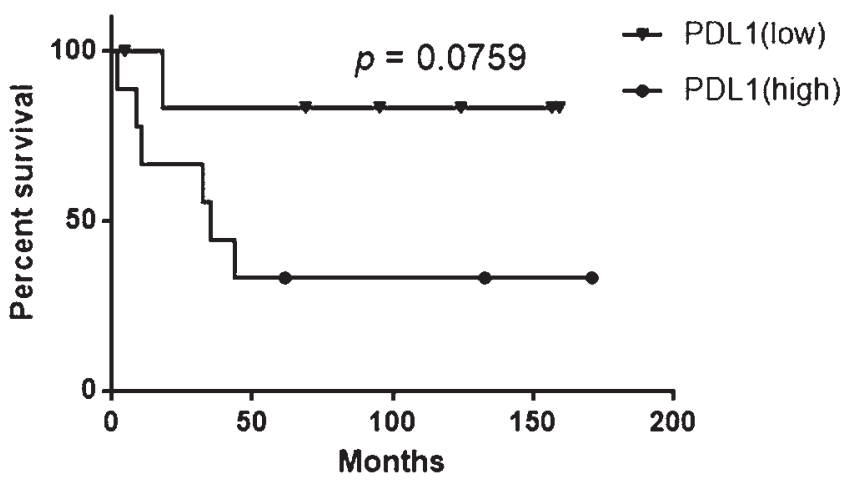

D

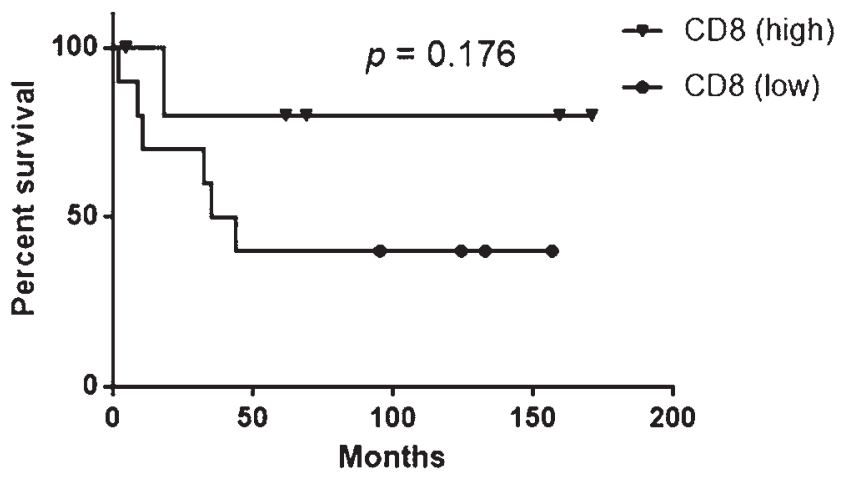

$\mathrm{F}$

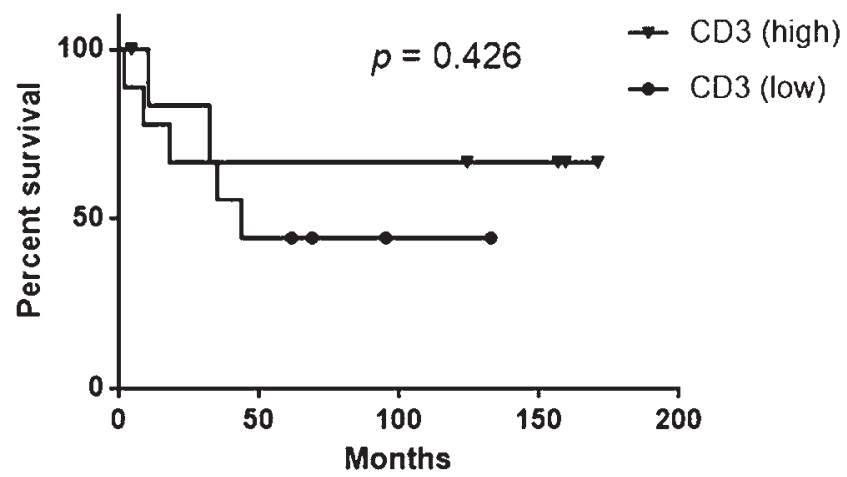

FIG. 2. Kaplan-Meier curves showing survival of patients with medulloblastoma stratified by PD-L1, CD3, and CD8 staining. The OS and PFS of patients with medulloblastoma was shown according to expression of PD-L1 (A and B) and the infiltration of CD8+ ( $C$ and $D)$ and CD3+ lymphocytes (E and F).

in preclinical and clinical trials. ${ }^{10}$ The Smoothened (SMO) inhibitor for $S H H$ subtypes with the $S M O$ mutation is currently being tested in clinical trials. ${ }^{3}$ Bromo and extra C-terminal (BET) bromodomain inhibitors, which interfere with $M Y C$-associated transcriptional activity, were reported to be effective in suppressing the growth of medulloblastoma cells with $M Y C$ amplification in vitro and in vivo. ${ }^{1,22}$ Because there is no established treatment for aggressive medulloblastoma, and radiotherapy for young children can cause cognitive impairment, the development of such multiple treatment modalities, including immuno- therapy, is essential to improve the prognosis of patients with medulloblastoma.

There is a limitation in our study. Because the number of patients in the study population is small, we could not perform reliable multivariate analysis in which covariates such as Ki-67 index, Chang's criteria, chemotherapy regimens, and radiation dosage were used. Besides, because the recruitment period of the study population was long ( $>22$ years), changes of diagnostic and treatment modalities might affect the prognosis of the patients. Because we have a small proportion of adult cases, the statistical 

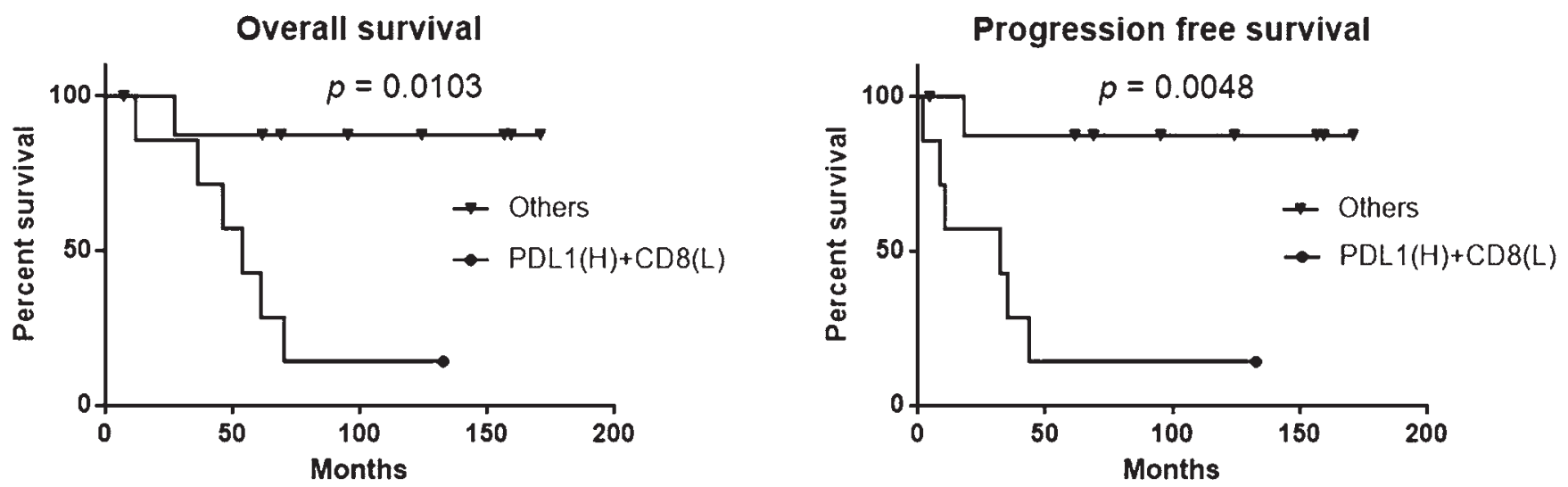

FIG. 3. Kaplan-Meier curves showing that high PD-L1 expression and low CD8+ lymphocyte infiltration (PDL1[H]+CD8[L]) was associated with worse survival in patients with medulloblastoma. The OS (left) and PFS (right) of patients with medulloblastoma was compared between tumors with high PD-L1 expression and low CD8+ lymphocyte infiltration and the other types of tumors.

power was not sufficient. Further large studies are needed to confirm our results.

\section{Conclusions}

Upregulation of PD-L1 expression with a reduced CD8+ lymphocyte infiltration was significantly associated with dismal prognosis in patients with medulloblastoma, and anti-PD-L1 antibody may be a potential treatment option for such a subgroup of patients. A larger study is necessary to confirm our results and to analyze the association between genetic signature and PD-L1 expression.

\section{Acknowledgments}

We thank the members in the Department of Neurosurgery for constructive comments on the manuscript and throughout the project. This work was supported by JSPS KAKENHI grants (Dr. Arakawa: Grants \#25462254 and \#21689039, Dr. Miyamoto: Grant \#25670623).

\section{References}

1. Bandopadhayay P, Bergthold G, Nguyen B, Schubert S, Gholamin S, Tang Y, et al: BET bromodomain inhibition of MYCamplified medulloblastoma. Clin Cancer Res 20:912-925, 2014

2. Choueiri TK, Fay AP, Gray KP, Callea M, Ho TH, Albiges L, et al: PD-L1 expression in nonclear-cell renal cell carcinoma. Ann Oncol 25:2178-2184, 2014

3. Gajjar AJ, Robinson GW: Medulloblastoma-translating discoveries from the bench to the bedside. Nat Rev Clin Oncol 11:714-722, 2014

4. Gerber NU, Mynarek M, von Hoff K, Friedrich C, Resch A, Rutkowski S: Recent developments and current concepts in medulloblastoma. Cancer Treat Rev 40:356-365, 2014

5. Hamanishi J, Mandai M, Iwasaki M, Okazaki T, Tanaka Y, Yamaguchi K, et al: Programmed cell death 1 ligand 1 and tumor-infiltrating $\mathrm{CD}^{+} \mathrm{T}$ lymphocytes are prognostic factors of human ovarian cancer. Proc Natl Acad Sci U S A 104:3360-3365, 2007

6. Kim JW, Eder JP: Prospects for targeting PD-1 and PD-L1 in various tumor types. Oncology (Williston Park) 28 (Suppl 3):15-28, 2014

7. Kluger HM, Zito CR, Barr ML, Baine MK, Chiang VL, Sznol M, et al: Characterization of PD-L1 expression and associated T-cell infiltrates in metastatic melanoma samples from variable anatomic sites. Clin Cancer Res 21:30523060, 2015

8. Larkin J, Chiarion-Sileni V, Gonzalez R, Grob JJ, Cowey CL, Lao CD, et al: Combined nivolumab and ipilimumab or monotherapy in untreated melanoma. N Engl J Med 373:2334, 2015

9. Liu Y, Carlsson R, Ambjørn M, Hasan M, Badn W, Darabi A, et al: PD-L1 expression by neurons nearby tumors indicates better prognosis in glioblastoma patients. J Neurosci 33:14231-14245, 2013

10. MacDonald TJ, Aguilera D, Castellino RC: The rationale for targeted therapies in medulloblastoma. Neuro Oncol 16:920, 2014

11. Northcott PA, Korshunov A, Witt H, Hielscher T, Eberhart CG, Mack S, et al: Medulloblastoma comprises four distinct molecular variants. J Clin Oncol 29:1408-1414, 2011

12. Okazaki T, Chikuma S, Iwai Y, Fagarasan S, Honjo T: A rheostat for immune responses: the unique properties of PD-1 and their advantages for clinical application. Nat Immunol 14:1212-1218, 2013

13. Okazaki T, Honjo T: The PD-1-PD-L pathway in immunological tolerance. Trends Immunol 27:195-201, 2006

14. Palmer SL, Reddick WE, Gajjar A: Understanding the cognitive impact on children who are treated for medulloblastoma. J Pediatr Psychol 32:1040-1049, 2007

15. Parsa AT, Waldron JS, Panner A, Crane CA, Parney IF, Barry JJ, et al: Loss of tumor suppressor PTEN function increases B7-H1 expression and immunoresistance in glioma. Nat Med 13:84-88, 2007

16. Pham CD, Flores C, Yang C, Pinheiro EM, Yearley JH, Sayour E, et al: Differential immune microenvironments and response to immune checkpoint blockade amongst molecular subtypes of murine medulloblastoma. Clin Cancer Res 22:582-595, 2016

17. Robert C, Long GV, Brady B, Dutriaux C, Maio M, Mortier $\mathrm{L}$, et al: Nivolumab in previously untreated melanoma without BRAF mutation. N Engl J Med 372:320-330, 2015

18. Schalper KA, Velcheti V, Carvajal D, Wimberly H, Brown J, Pusztai L, et al: In situ tumor PD-L1 mRNA expression is associated with increased TILs and better outcome in breast carcinomas. Clin Cancer Res 20:2773-2782, 2014

19. Shih DJH, Northcott PA, Remke M, Korshunov A, Ramaswamy V, Kool M, et al: Cytogenetic prognostication within medulloblastoma subgroups. J Clin Oncol 32:886-896, 2014

20. Topalian SL, Hodi FS, Brahmer JR, Gettinger SN, Smith DC, McDermott DF, et al: Safety, activity, and immune correlates 
of anti-PD-1 antibody in cancer. N Engl J Med 366:24432454, 2012

21. Varghese F, Bukhari AB, Malhotra R, De A: IHC Profiler: an open source plugin for the quantitative evaluation and automated scoring of immunohistochemistry images of human tissue samples. PLoS One 9:e96801, 2014

22. Venkataraman S, Alimova I, Balakrishnan I, Harris P, Birks DK, Griesinger A, et al: Inhibition of BRD4 attenuates tumor cell self-renewal and suppresses stem cell signaling in MYC driven medulloblastoma. Oncotarget 5:2355-2371, 2014

23. Wang A, Wang HY, Liu Y, Zhao MC, Zhang HJ, Lu ZY, et al: The prognostic value of PD-L1 expression for non-small cell lung cancer patients: a meta-analysis. Eur J Surg Oncol 41:450-456, 2015

24. Weber JS, D’Angelo SP, Minor D, Hodi FS, Gutzmer R, Neyns B, et al: Nivolumab versus chemotherapy in patients with advanced melanoma who progressed after anti-CTLA-4 treatment (CheckMate 037): a randomised, controlled, openlabel, phase 3 trial. Lancet Oncol 16:375-384, 2015

\section{Disclosures}

The authors report no conflict of interest concerning the materi- als or methods used in this study or the findings specified in this paper.

\section{Author Contributions}

Conception and design: Arakawa, Murata, Fukui. Acquisition of data: Murata, Fujimoto, Terada, Yokogawa, Minamiguchi, Yamaguchi. Analysis and interpretation of data: Murata, Mineharu, Tanji, Fujimoto, Yokogawa. Drafting the article: Arakawa, Murata, Mineharu. Critically revising the article: Arakawa, Mineharu, Liu, Tanji, Yamaguchi, Fujimoto, Fukui, Terada, Yamaguchi. Reviewed submitted version of manuscript: Liu, Miyamoto. Approved the final version of the manuscript on behalf of all authors: Arakawa. Statistical analysis: Murata, Mineharu. Administrative/technical/material support: Yamaguchi, Miyamoto. Study supervision: Miyamoto.

\section{Correspondence}

Yoshiki Arakawa, Department of Neurosurgery, Kyoto University Graduate School of Medicine, 54 Kawahara-cho, Shogoin, Sakyo-ku, Kyoto 606-8507, Japan. email: yarakawa@kuhp. kyoto-u.ac.jp. 